\title{
Springtime migratory restlessness and departure orientation of Great Knots (Calidris tenuirostris) in the south compared to the north Yellow Sea
}

\author{
Ning Hua', Susanne Åkesson², Qianyan Zhou' and Zhijun Ma ${ }^{1 *}$
}

\begin{abstract}
Background: The motivation of birds to proceed with migration is associated with both endogenous and exogenous factors. According to their migratory situation and to the characteristics of stopover sites, birds might exhibit migratory motivation differently among sites. Although migratory motivation of migrating birds has been well studied in many species, the investigation of the same species in different migratory situations and at different stopover sites is still limited. We predicted that birds at different stopover sites could differ in migratory disposition, including expression of migratory restlessness and responses to environmental cues.
\end{abstract}

Methods: Here we compared migration motivation and orientation of Great Knots (Calidris tenuirostris) at two stopover sites, Chongming Dongtan in the south Yellow Sea, which is a temporary rest site, and Yalujiang Estuary Wetland in the north Yellow Sea, which is a critical refuelling site, during northward migration. Modified Emlen funnels, with thermal paper inside to record scratches of the birds, were used to detect activity (intensity and direction) of birds. Environmental conditions, including wind direction and speed, cloud cover, tide condition, times of the day (before and after sunset) were recorded during experiments. Generalized linear models were used to detect the effects of endogenous and exogenous factors on the migratory motivation of the birds.

Results: In the south Yellow Sea, the migratory activity intensity of Great Knots was positively related to wind assistance. In the north Yellow Sea, where birds exhibited higher intensity of migratory activity than in the south, the intensity increased and the motivation to initiate migration grew stronger as the season advanced. The Great Knots exhibited wind-related orientation behaviour in the funnels at both sites.

Conclusions: The results suggest that the intensity of migratory motivation differed between the two sites. Departure decisions of Great Knots appear to be affected mainly by external factors at the temporary rest site in the south Yellow Sea but possibly by endogenous factors at the final pre-breeding refuelling site in the north Yellow Sea.

Keywords: Migratory restlessness, Orientation, Departure decision, Migratory situation, Spatio-temporal program, Stopover, Yellow Sea, Shorebirds

\footnotetext{
*Correspondence: zhijunm@fudan.edu.cn

${ }^{1}$ Coastal Ecosystems Research Station of Yangtze River Estuary, Ministry

of Education Key Laboratory for Biodiversity Science and Ecological

Engineering, Institute of Biodiversity Science, Fudan University,

Shanghai 200433, China

Full list of author information is available at the end of the article
} 


\section{Background}

Migratory birds need one or more sites to rest or to refuel during their journey, and their decision to continue to stop or resume migration is related to both endogenous and external factors (Newton 2008). As indicators of the motivation to proceed with migration, migratory restlessness and its directional preference have been associated with various factors, such as seasonality and time of the day, body condition of the birds, position of the stopover site, and weather condition such as wind assistance and cloud cover of the sky (Sandberg et al. 1988; Able 1991; Åkesson and Sandberg 1994; Sandberg 2003; Deutschlander and Muheim 2009; for review, see Newton 2008). With respect to shorebirds living along the coasts, tidal regime has also been reported to be associated with the migration departure behaviours (Piersma et al. 1990; Tulp et al. 1994). According to their migratory situation and to the characteristics of stopover sites, birds might exhibit migratory motivation differently among sites, i.e., the relationship between migratory restlessness and various factors may differ among sites. For example, energetic condition usually takes precedence when birds are confronted with an ecological barrier, thus they will suppress migratory restlessness and even perform temporary reverse migration unless enough fuel is stored (Åkesson et al. 1996a; Åkesson 1999; Sandberg et al. 2002). However, at sites with refuelling possibilities ahead closely, migratory motivation of birds might not be necessarily associated with fuel stores (Åkesson 1999; Tsvey et al. 2007). Although migratory motivation in the form of migratory restlessness has been well studied in many species, the investigation of the same species in different migratory situations and at different stopover sites along the same migratory route is still limited.

The stopover behaviour of the Great Knot (Calidris tenuirostris) within the Yellow Sea during spring migration provides a possibility to study its diversity of expressing migratory motivation at different sites along the same route. The Great Knot is a mediumsized shorebird that performs long-distance migratory flights between their major nonbreeding sites in Northwest Australia and breeding sites in East Siberia. During northward migration, the presence of the Great Knot population in the Yellow Sea region lasts for nearly 2 months, where they refuel before flying to the breeding area (Ma et al. 2013; Bai et al. 2015). The Yangtze estuary in the south Yellow Sea (Fig. 1) is the first major stopover site for some Great Knots after a trans-Pacific flight of over $5000 \mathrm{~km}$ initiating from the nonbreeding sites (Battley et al. 2000; Lisovski et al. 2016). The coast along the Yalu Jiang estuary in the north Yellow Sea is one of the last refuelling sites before the final non-stop flight of over $4000 \mathrm{~km}$ to the breeding sites (Bamford et al. 2008;

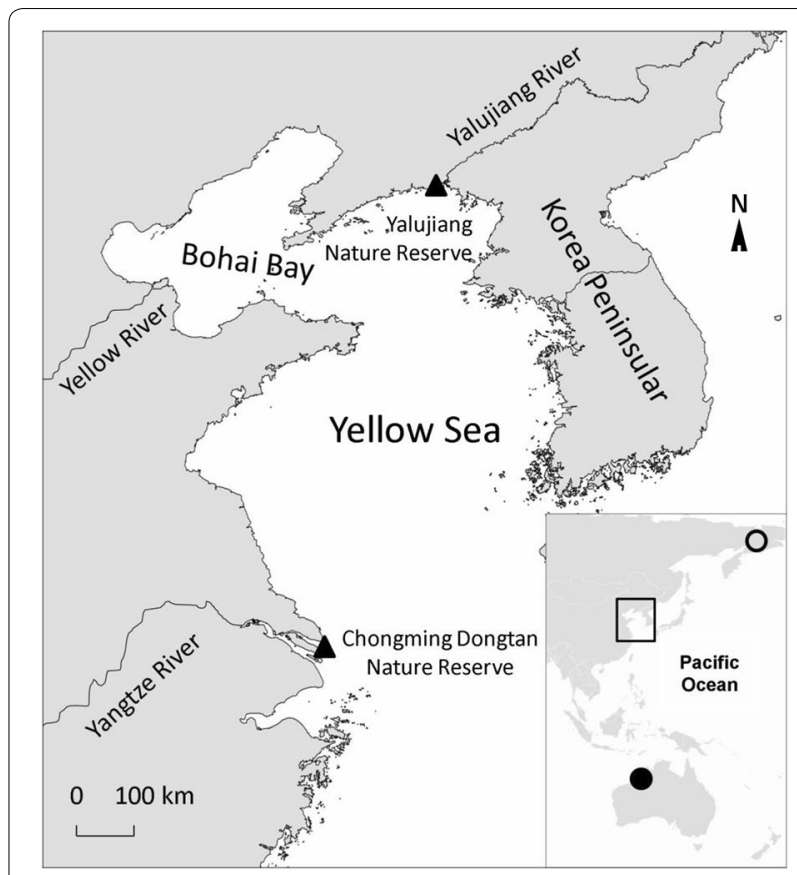

Fig. 1 Locations of the Chongming Dongtan Nature Reserve (CMDT, filled triangle) and the Yalujiang Estuarine Wetland Nature Reserve (YLE, filled triangle) in the Yellow Sea. The inset map shows the locations of the Yellow Sea (square) and the main breeding area (hollow circle) in east Siberia and the nonbreeding area (filled circle) in northwest Australia of Great Knots along the East Asian-Australasian Flyway

Ma et al. 2013; Lisovski et al. 2016). There are large areas of suitable habitats for Great Knots between the south and the north Yellow Sea but few between the north Yellow Sea and the breeding sites. Individuals of Great Knots remain in the south Yellow Sea for only a short period (average 2 days) and store little fuel before moving to the north Yellow Sea (Ma et al. 2013; Peng et al. 2015). Individuals in the north Yellow Sea, in contrast, stay for about 1 month on average and deposit sufficient fuel reserves for the much longer non-stop flight to the breeding sites (Ma et al. 2013; Peng et al. 2015). Departures of Great Knots in the south Yellow Sea extend over a long time period, and the departure date is unsynchronized among individuals but is related to wind conditions (Ma et al. 2011). Contrastingly, the departure date of Great Knots in the north Yellow Sea is highly synchronized, with most birds leaving within a 1-week period, suggesting an intrinsic regulation by spatio-temporal programme (Ma et al. 2013). Such differences in stopover duration, refuelling, and departures indicate that, relative to birds in the south Yellow Sea, those in the north Yellow Sea may experience a more intense urge for migration because of the longer flight distance, the higher energetic requirement, and the more serious time 
constraint. Given such different migratory situations in the south and the north Yellow Sea, we expect that birds at the two stopover sites will differ in migratory disposition including expression of migratory restlessness and responses to environmental cues.

Here we investigated migratory motivation of Great Knots and its relationship with a series of factors by orientation experiments at two stopover sites in the Yellow Sea during northward migration. We predicted that (1) birds in the north Yellow Sea would express stronger migratory motivation than birds in the south Yellow Sea, with stronger migratory restlessness and/or more concentrated seasonal appropriate directional preference; (2) the intensity of motivation to depart from the south Yellow Sea might be related to external environmental factors such as wind assistance; (3) the intensity of motivation to initiate migration in the north Yellow Sea could possibly be related to the date as a result of endogenous regulation, and might be associated with body condition since large amount of fuel stores are critical for the subsequent long-distance migratory flight.

\section{Methods}

Study sites

Fieldwork was conducted at the Chongming Dongtan Nature Reserve (CMDT, $\left.31.5^{\circ} \mathrm{N}, 121.9^{\circ} \mathrm{E}\right)$ in the south Yellow Sea and at the Yalujiang Estuarine Wetland Nature Reserve (YLE, $39.8^{\circ} \mathrm{N}, 123.9^{\circ} \mathrm{E}$ ) in the north Yellow Sea (Fig. 1). There are semidiurnal tidal fluctuations in the Yellow Sea region, with two distinct cycles of ebb and flow tides each day (Ma et al. 2011, 2013). Great Knots generally stay on the mudflats unless they are submerged by tidewater. The presence of Great Knots lasts for 1 month (from late March to late April) at CMDT and for nearly 2 months (from late March to mid-May) at YLE (Ma et al. 2011, 2013). During northward migration, about $30 \%$ of the Great Knots stayed at CMDT would use YLE later in the same season (Ma et al. 2013).

\section{Experimental procedure}

Orientation tests were conducted during the northward migration from 2010 to 2012. All fieldwork was conducted with the permission and support of the local nature reserves. Great Knots were captured on the intertidal flat with clapnets as part of the shorebird banding program (Choi et al. 2009). We measured body mass (to the nearest $0.1 \mathrm{~g}$ ) and took biometrics of birds shortly after capture. The age of the birds was determined according to their plumage characteristics (Prater et al. 1977). We randomly selected up to nine adults per day and temporally kept them in an outdoor aviary $(3 \mathrm{~m} \times 2 \mathrm{~m} \times 2 \mathrm{~m}$, made of bamboo frame and nylon nets) near the capture sites before orientation tests. The aviaries were exposed to the natural photoperiod, ambient temperature and wind, and local magnetic field but were protected from human disturbance, thus gave the birds an opportunity to perceive environmental cues (e.g., tide cycle, Gudmundsson and Sandberg 2000) and to potentially determine whether and which direction to depart. We supplied unlimited water and food (small shrimps and bivalves) to birds in captivity although only some of them would actively feed. All of the selected birds were used for orientation tests only on the capture day and were released immediately after the tests. While kept the birds lost $2.4 \%$ of body mass on average (SD $=3.4 \%$ ), which would unlikely have any adverse influence on their migration or survival because the Great Knots regularly lose about $50 \%$ of body mass when making long-distance flights (Battley et al. 2000).

We used modified Emlen funnels (Emlen and Emlen 1966; Sandberg and Gudmundsson 1996) covered with thermal paper inside to record scratches of the birds (Mouritsen et al. 2009). The funnels were made of nonmagnetic materials (thin wood board) with a top diameter of $650 \mathrm{~mm}$, a bottom diameter of $250 \mathrm{~mm}$, and a height of $350 \mathrm{~mm}$. The top of each funnel was covered with a fine-mesh nylon net, giving the standing Great Knots approximately $130^{\circ}$ visual access to the natural sky. Thus birds inside the funnels could detect the movement of cloud, which could be used as a cue of wind direction (Åkesson and Hedenström 2000). The orientation tests were performed under natural sky in the local geomagnetic field. The funnels (up to nine in each experiment) were placed on flat and open ground near the capture sites on intertidal flat, with intervals of at least $3 \mathrm{~m}$. All the experimental birds were weighed again just before the tests began. Each test lasted for $1 \mathrm{~h}$. We recorded the number and distribution of the scratches left by bird claws to assess their migratory restlessness and orientation performance (Mouritsen et al. 2009).

Generally migratory departure is preferred during a rising or high tide in the late afternoon before sunset in shorebirds including Great Knots, and they migrate throughout the night (Alerstam et al. 1990; Piersma et al. 1990; Tulp et al. 1994). To record the migratory restlessness of Great Knots when they are probably most motivated to depart, and to record possible temporal variation in migratory restlessness, orientation tests were conducted at two time periods of the day: before and after sunset. Tests before sunset were conducted in 2011 at CMDT and in 2012 at YLE; these tests began in the late afternoon and ended before the sun elevation angle reached $0^{\circ}$. Tests after sunset were carried out at both sites in 2010 and began at least $1 \mathrm{~h}$ after sunset. Given the possible influence of tidal regime on migratory motivation of the Great Knots, we recorded tide 
conditions at the middle of the tests as a "HW" (within the tide window of high water $\pm 250 \mathrm{~min}$ ) and a "LW" (outside the tide window) (Gudmundsson and Sandberg 2000). Because different orientation performances by migrating birds have been observed under clear versus overcast skies (Richardson 1982; Sandberg et al. 1988; Gudmundsson and Sandberg 2000; Grönroos et al. 2010), and field studies have indicated that wind condition near the ground level can also affect the motivation to initiate migration ( $\AA$ kesson and Hedenström 2000; Ma et al. 2011), cloud cover and wind condition during the test hour were recorded. Cloud cover was evaluated according to the records at the beginning, the middle, and the end of each test ( $0 / 8$ : cloudless; $8 / 8$ totally overcast), as clear $(0 / 8-3 / 8)$, partly cloudy $(4 / 8-6 / 8)$, and overcast $(7 / 8-8 / 8)$ skies. Wind direction at ground level during every test was recorded as one of 16 compass directions, and wind speed was estimated by a handheld anemometer (Kestrel 4000). Wind condition changed marginally throughout the whole hour of a test.

\section{Data analysis}

We used the total number of the scratches as an estimation of the intensity of migratory activity. We visually estimated the median direction to the nearest $5^{\circ}$ to determine the orientation of each bird (Mouritsen 1998). A concentration score was also given following Grönroos et al. (2010) according to the angle within which the median direction without doubt lies $\left(0:>45^{\circ}, 1: 21^{\circ}-45^{\circ}\right.$, 2: $11^{\circ}-20^{\circ}, 3: 6^{\circ}-10^{\circ}, 4: 0^{\circ}-5^{\circ}$ ). In a total of 295 samples, there were eight individuals whose scratches had a bimodal distribution. Hereinto, for seven individuals, direction with more scratches was considered the preferred direction and was used in subsequent analysis, and the remained individual whose scratches were equally distributed in two directions was considered disoriented and was not included in analysis of direction preference. Scratches were independently assessed by two persons ( $\mathrm{N}$ Hua and Q Zhou), and the assessments of the two observers were always similar. Birds that left fewer than 20 scratches were considered inactive, and birds with a concentration score of 0 were considered disorientated; these records were excluded from the orientation analysis (Table 1). All the records were included in the analysis of activity.

Wind effects $(\Delta W)$ on bird migration were calculated following Piersma and Jukema (1990): $\Delta W=W \times \cos \alpha$ $+\left(A^{2}-(W \times \sin \alpha)^{2}\right)^{0.5}-A$, where $W$ was the wind speed $(\mathrm{m} / \mathrm{s})$ at ground level, $\alpha$ was the angular difference between the wind direction (from which direction the wind was blowing, $w$ ) and the migration direction $(t), \alpha=w \pm 180^{\circ}-t$, and $A$ was the airspeed of migrating birds (assumed to average $18 \mathrm{~m} / \mathrm{s}$ following Battley
Table 1 Number of Great Knots in orientation tests at Chongming Dongtan Nature Reserve (CMDT) in the south Yellow Sea and at Yalujiang Estuarine Wetland Nature Reserve (YLE) in the north Yellow Sea during northward migration

\begin{tabular}{llllll}
\hline Site & Time period & \multicolumn{4}{c}{ Number (and percentages) of Great Knots } \\
\cline { 3 - 6 } & & Inactive & Disoriented & Oriented & Total \\
\hline \multirow{2}{*}{ CMDT } & Before sunset & 46 & 10 & $89(61.4 \%)$ & 145 \\
& After sunset & 41 & 0 & $37(47.4 \%)$ & 78 \\
YLE & Before sunset & 5 & 5 & $23(69.7 \%)$ & 33 \\
& After sunset & 15 & 1 & $23(59.0 \%)$ & 39 \\
Total & & $36.3 \%$ & $5.4 \%$ & $58.3 \%$ & \\
\hline
\end{tabular}

1997). The positive value of wind effect means tailwind while negative value headwind. The larger the value, the stronger the wind along the flight direction. We visually estimated the centre of the breeding sites according to the breeding distribution map of Great Knots (Bamford et al. 2008). Along the great circle path, the migration direction of Great Knots is $10.5^{\circ}$ at CMDT (towards YLE, consistent with an observed mean departure direction of $11^{\circ}$ in Ma et al. 2011) and $37.5^{\circ}$ at YLE (towards the centre of the breeding sites).

We used body mass to indicate the body condition of each bird. The deviation of body mass at capture and at test was calculated as "body mass decrease" to indicate change in body condition during captive period.

Generalized linear models (GLM) was used to test the effects of times of the day (before sunset and after sunset), sites (CMDT and YLE), and their interactions on the intensity of migratory activity of Great Knots. Because over-dispersion was detected in the count data, quasiPoisson GLM was used to analyse the data. At both sites the mean scratch number recorded after sunset declined over $80 \%$ compared to that before sunset, and thus might be less representative of migratory motivation (see "Discussion" section). Therefore, with the two data subsets (two sites) before sunset, we further used generalized linear models to detect the effects of bird condition (body mass at capture, body mass decrease), date, weather condition (cloud cover, wind effect), and tide condition on the activity intensity of birds. Due to limitation of sample size, we did not involve interactions of variables in the models. The second-order-corrected Akaike's information criterion $\left(\mathrm{AIC}_{\mathrm{c}}\right.$, Burnham and Anderson 2002) was used to select the optimal models among all the combinations. The model with the smallest $\mathrm{AIC}_{\mathrm{c}}$ and the largest weight was considered the best explanatory model. However, other models with delta $\mathrm{AIC}_{\mathrm{c}}<2$ were considered equally plausible in light of the data (Burnham and Anderson 2002). The average model coefficients for the 
variables involved in the competitive models were calculated according to their beta estimates and AIC weights of each model (Burnham and Anderson 2002). Parameters with a significant estimate in the optimal model were considered to influence the intensity of migratory restlessness.

The sample mean vector of experimental birds was calculated for each of the four data subsets (two sites $\times$ two times) according to standard procedures (Batschelet 1981). The Rayleigh test was used to determine whether the mean of the individual median directions of every data subset (hereafter the natural heading) was significantly different from random. The sun azimuth at the middle of each test was calculated by the NOAA Solar Calculator (available at: http://www.esrl.noaa.gov/gmd/ $\mathrm{grad} /$ solcalc/). The mean direction towards the sun was calculated for each data subsets. To investigate the relationship between the orientation of birds and the wind direction, we recalculated the median direction of each bird in the funnel with the corresponding wind direction setting at $0^{\circ}$, as a shifted direction. The mean of the shifted individual median directions was calculated for each data subset, as the shifted heading hereafter. The consistency of migration direction, or sun direction, with the natural heading of the birds was accepted or rejected depending on whether it was within or outside the $95 \%$ confidence interval of the mean direction. The consistency of wind direction with the shifted heading of the birds was accepted or rejected depending on whether it was within or outside the $95 \%$ confidence interval of the mean of the shifted directions.

Circular data were statistically analysed using Oriana (Ver. 4.01, Kovach Computing Services), and non-circular data were analysed using $\mathrm{R}$ (Ver. 2.15.0).

\section{Results}

\section{Intensity of migratory activity}

Quasi-Poisson regression indicated that the intensity of migratory activity of Great Knots was significantly affected by the times of the day and the site (Table 2). The activity intensity after sunset was significantly lower than

Table 2 Estimated coefficients of the variables and their correspondent $t$ value for predicting the migratory activity intensity of Great Knots using quasi-Poisson GLM model

\begin{tabular}{lcrl}
\hline Parameter & Estimate & $\boldsymbol{t}$ value & $\boldsymbol{p}$ value \\
\hline Intercept & 5.33 & 36.78 & $<0.001^{* * *}$ \\
Times of the day (after sunset) & -0.83 & -2.49 & $0.01^{*}$ \\
Sites (YLE) & 0.61 & 2.28 & $0.02^{*}$ \\
Interaction & 0.21 & 0.43 & 0.67
\end{tabular}

*** $p<0.001 ; * p=0.01-0.05$ that before sunset; birds exhibited higher activity intensity at YLE than at CMDT (Fig. 2). The interaction of times of the day and sites did not significantly affect the activity intensity (Table 2), suggesting that at both sites, the Great Knots demonstrated higher activity intensity before sunset than after sunset, and that in both time periods, they exhibited higher activity intensity at YLE than at CMDT.

Factors associated with intensity of migratory activity before sunset differed between sites. At CMDT, the intensity of migratory activity was closely related to the wind effect, which was involved in all the candidate models (Additional file 1: Table S1). Activity intensity significantly increased with the increase of wind assistance (Table 3; Fig. 3a). Moreover, variables of date, body mass, and body mass decrease were also involved in the candidate models (Table 3, Additional file 1: Table S1). At YLE, the most significant factor associated with the activity intensity was the date, which was involved in the best three models predicting activity intensity (Additional file 1: Table S1), i.e., intensity increased as the migration season progressed (Table 3; Fig. 3b). Activity intensity at YLE was also negatively associated with body mass decrease (Fig. 3c) and wind effect (Fig. 3d). Moreover, variables of body mass and tide were also involved in the candidate models (Table 3, Additional file 1: Table S1).

\section{Orientation}

In tests before sunset (Additional file 1: Table S2), Great Knots exhibited a significant unimodal directional preference, and their average natural headings were towards

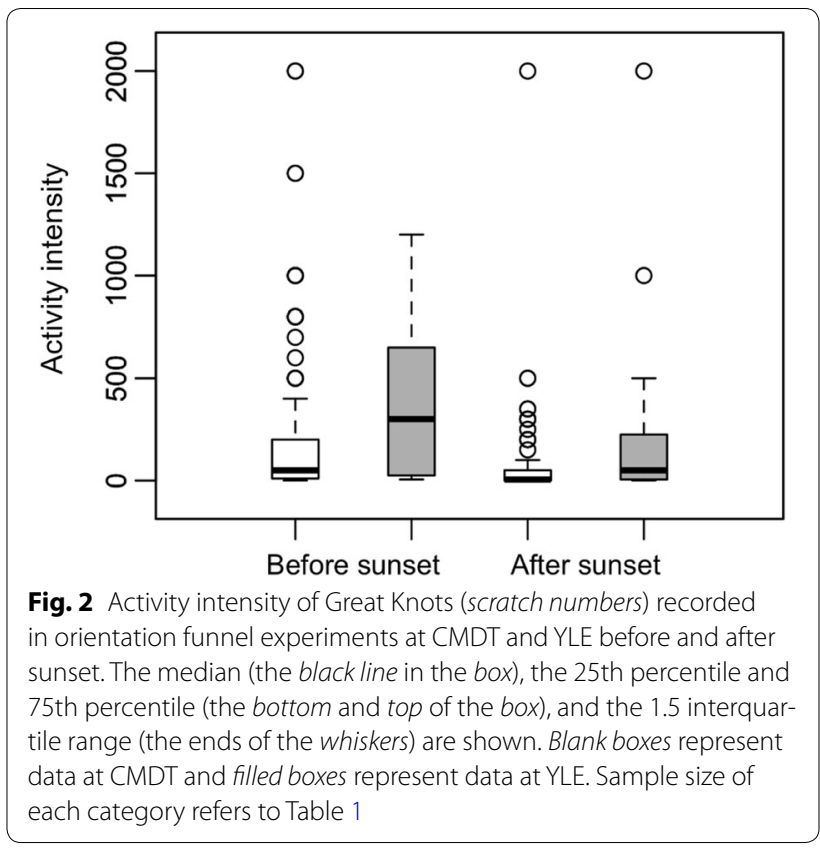


Table 3 Summary of the optimal models predicting the activity intensity of Great Knots before sunset based on data from orientation funnels at two stopover sites (CMDT in the south Yellow Sea and YLE in the north Yellow Sea)

\begin{tabular}{|c|c|c|c|c|c|c|c|c|c|c|c|}
\hline Site & Intercept & Wind effect & Date & Body mass decrease & Body mass & Tide & Cloud & $n$ & $R^{2}$ & $F$ & $p$ \\
\hline CMDT & $1.74 \pm 0.14^{* * *}$ & $0.02 \pm 0.00^{*}$ & $0.00 \pm 0.00$ & $-0.00 \pm 0.01$ & $0.00 \pm 0.00$ & - & - & 145 & 0.03 & 4.67 & 0.032 \\
\hline YLE & $1.89 \pm 0.31^{* * *}$ & $-0.05 \pm 0.07^{*}$ & $0.02 \pm 0.02^{* *}$ & $-0.02 \pm 0.03^{*}$ & $0.00 \pm 0.00$ & $0.49 \pm 0.54$ & - & 33 & 0.23 & 2.89 & 0.053 \\
\hline
\end{tabular}

The estimated coefficients (mean $\pm \mathrm{SE}$ ) for the variables were calculated according to their beta estimates and AIC weights of the competitive models (Additional file 1: Table S1) (Burnham and Anderson 2002). The significant levels for the estimated coefficients of the included parameters are listed (***<0.001; ${ }^{* *} 0.001-0.01$; *0.01-0.05; - not involved)

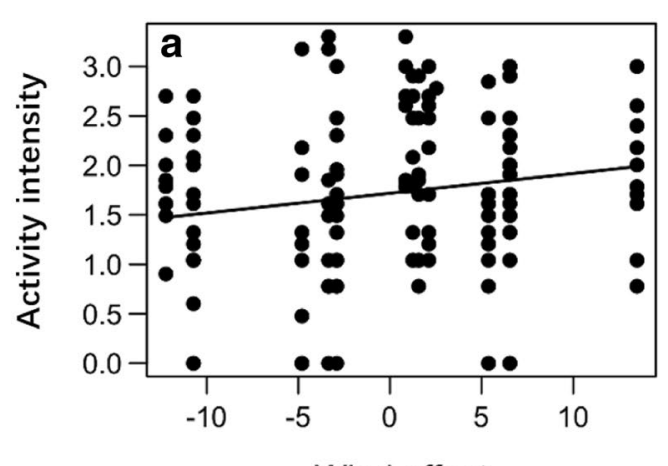

Wind effect

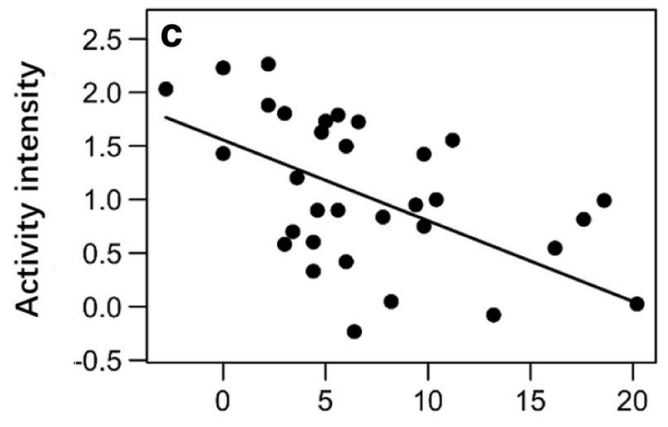

Body mass decrease $(\mathrm{g})$
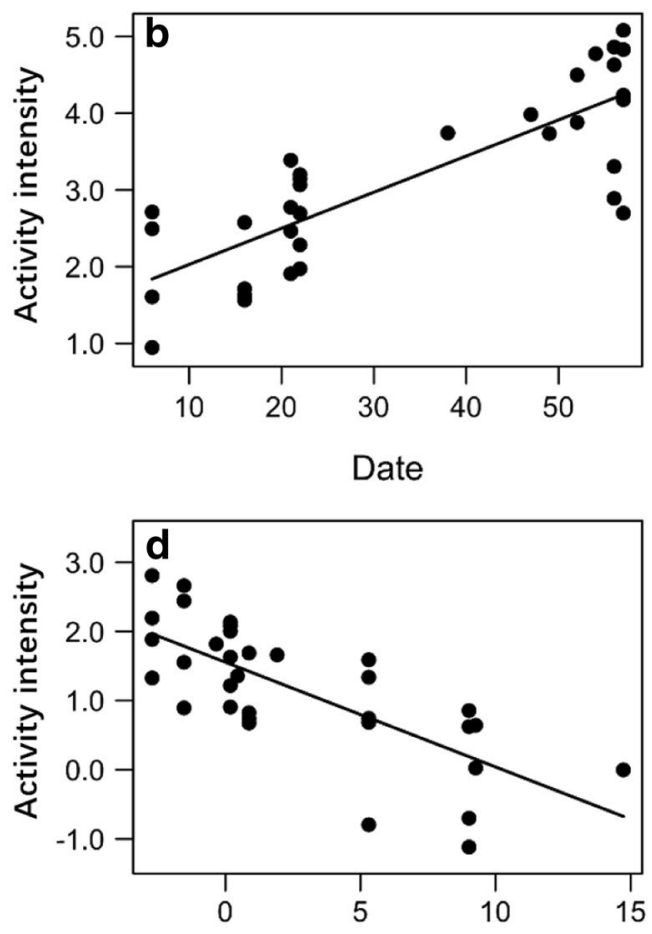

Wind effect

Fig. 3 Factors significantly related to the activity intensity (log-transformed scratch numbers) of Great Knots recorded in orientation funnel cages before sunset at CMDT and YLE. According to the $\mathrm{AIC}_{c}$ value of all the candidate models (Additional file 1: Table S1), the best model for bird activity intensity includes only the wind effect at CMDT (a) while includes test date (b), body mass decrease (c), and wind effect (d) at YLE. Adjusted activity intensity is shown for data at YLE to exclude the influence of factors other than the presented one. Additional details are provided in Table 3

SE at both sites (Fig. 4a, c); neither the migration direction nor the average sun azimuth was within the 95\% confidence interval of the average natural headings of the tested birds. Birds did not indicate a significant directional preference after sunset (Fig. 4b, d). Moreover, there were no significant differences in the distribution of natural headings between the two sites either before sunset (Watson's $U^{2}$ test, $U=0.054, p>0.5$ ) or after sunset $(U=0.051, p>0.5)$ or between the two time periods at CMDT $(U=0.092, p>0.2)$ or YLE $(U=0.083$, $p>0.2$ ).

After we recalculated the orientation relative to the corresponding wind direction at the time of the test
(Additional file 1: Table S3), the average direction of all data subsets became different from random (suggested different from random at CMDT after sunset, $p=0.079$ ). Wind direction $\left(0^{\circ}\right)$ was always within the $95 \%$ confidence interval, except for tests at YLE before sunset, which included wind direction in the $99 \%$ confidence interval of the average shifted bird heading (Fig. 5). Compared to the natural headings, the length of the mean vector and $p$ value of the shifted directions increased in each data subset. The distribution of shifted directions did not significantly differ between test times at CMDT (Watson's $U^{2}$ test, $\left.U=0.097, p>0.2\right)$ or at $\mathrm{YLE}(U=0.019, p>0.5)$, but did significantly differ between the two sites before 

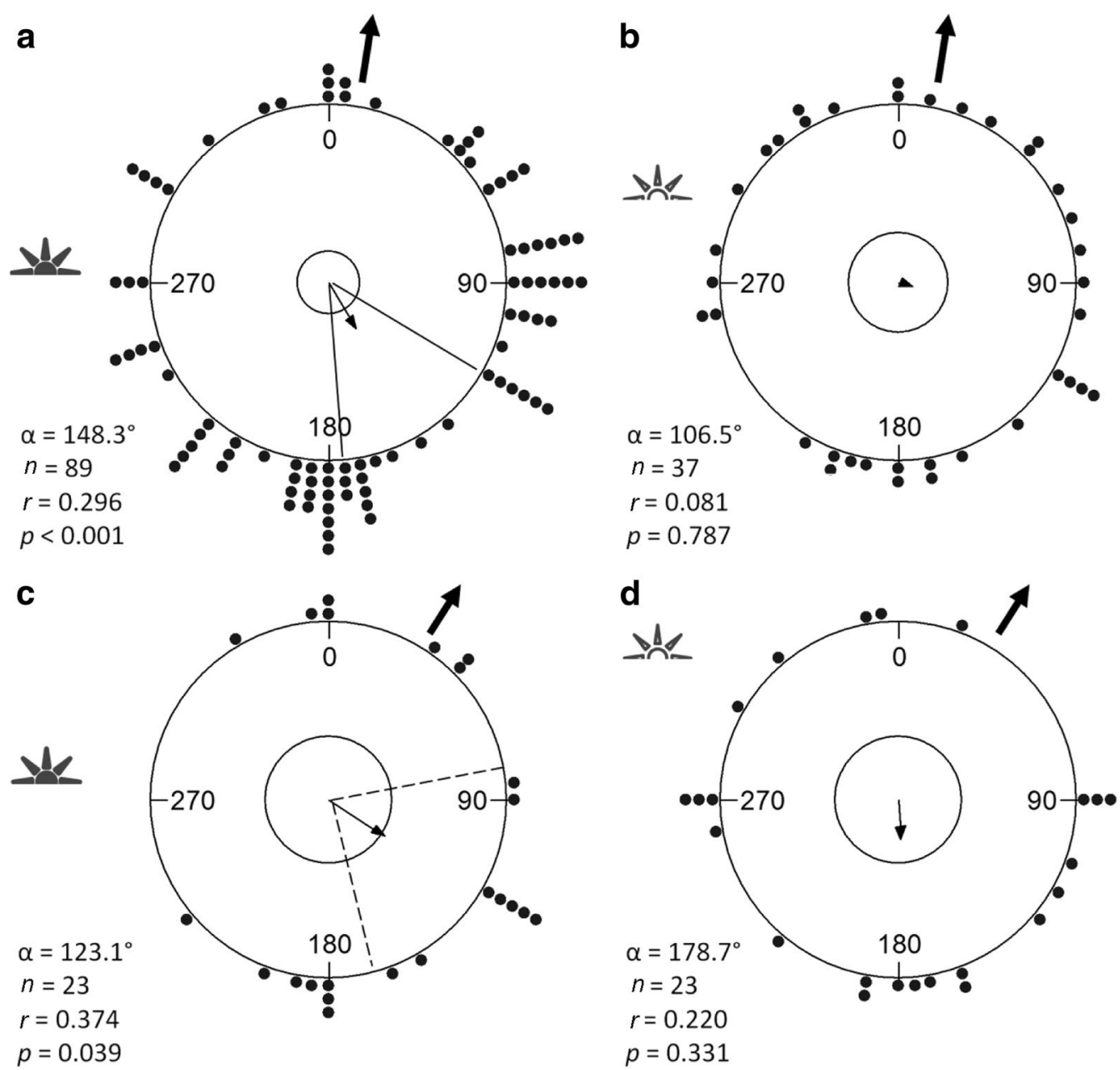

Fig. 4 Natural headings of the Great Knots at CMDT before (a) and after sunset (b) and at YLE before (c) and after sunset (d). Each filled dot shows the direction of an individual bird. The average direction ( $a$, arrow at the center of the circle), sample size $(n)$, mean vector length $(r$, length of the arrow), and $p$ value of the Rayleigh test are indicated. The $95 \%$ confidence interval (lines beside the arrow) is presented only when the $p$ value is $<0.05$. A dashed line in (c) indicates that the confidence interval is unreliable because of small sample size or low concentration. The mean direction of the sun is indicated by a sun icon, and the migration direction at CMDT and YLE is indicated by a bold arrow outside the circle in each panel

sunset $(U=0.232, p<0.02)$ and after sunset $(U=0.204$, $p<0.05)$.

\section{Discussion}

\section{Intensity of migratory activity}

We found that the Great Knots in the funnels exhibited a temporal variation in their migratory restlessness, which might correspond with their daily timing of migration initiation. Shorebirds tend to start migration a few hours before sunset during a rising or high tide (Alerstam et al. 1990; Piersma et al. 1990), and the same pattern has been observed for Great Knots in the field (Tulp et al. 1994). This explains the lower intensity of activity after sunset than before sunset at both stopover sites in this study. Temporal variation of activity intensity has also been reported in orientation experiments with passerines, with high intensity at sunset and sunrise but reduced intensity at night (Åkesson and Sandberg 1994). These results indicate that migratory birds are more active when information concerning the sun position or skylight polarization is available (Åkesson et al. 1996b) because the sun compass might be involved in migration of both passerines and shorebirds (Åkesson and Bäckman 1999; Muheim et al. 2003; Grönroos et al. 2010). As a consequence, activity before sunset might be more related to the migration decision, while this relationship may not be as pronounced when it turns dark although birds could still retain some motivation to migrate (Åkesson et al. 1996b).

The tested birds were more active at YLE than at CMDT both before and after sunset. This suggests that the migratory motivation of the Great Knots increased as 

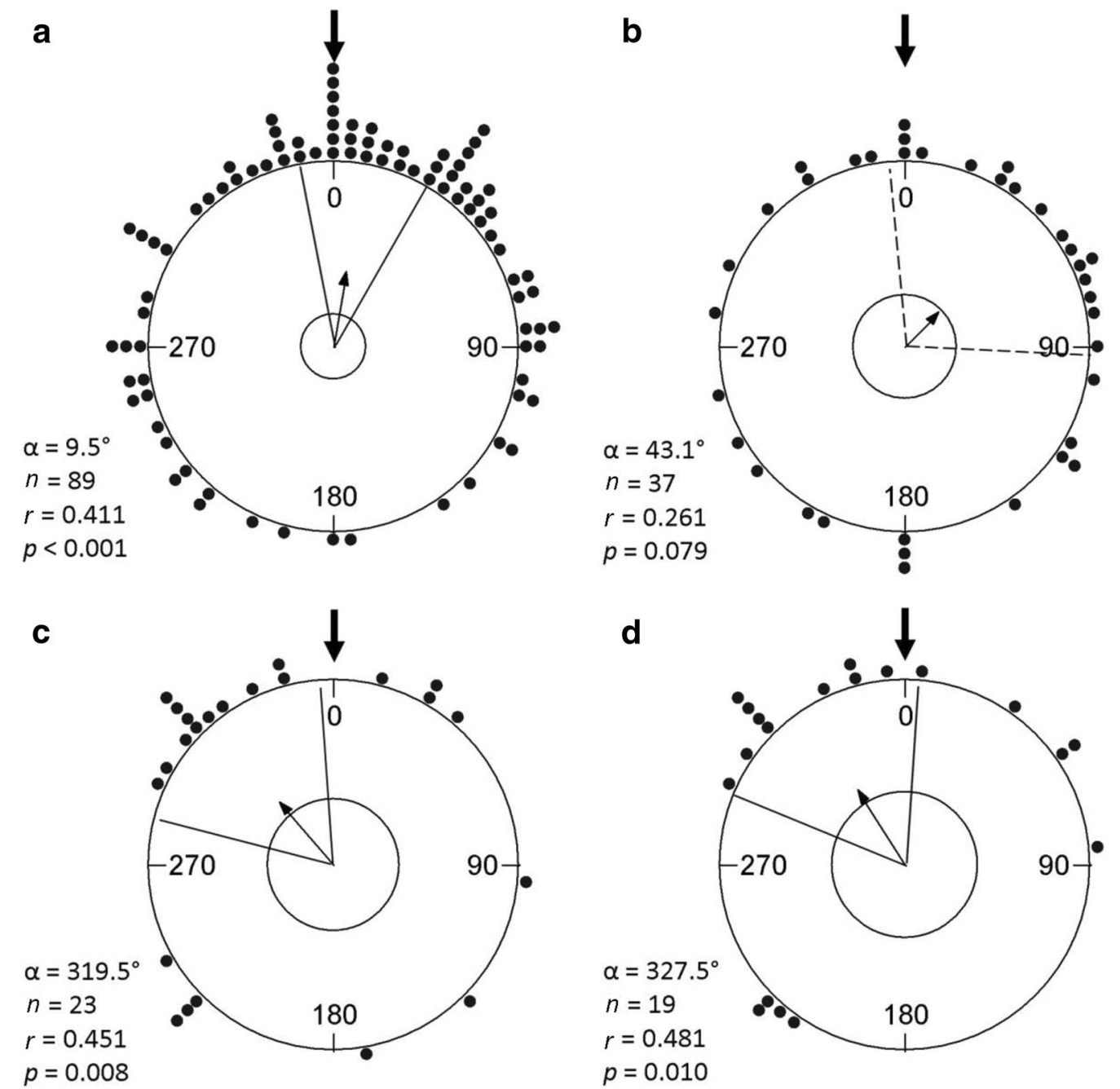

Fig. 5 Shifted headings of birds when the wind direction is considered as $0^{\circ}$ at CMDT before (a) and after sunset (b) and at YLE before (c) and after sunset (d). Each filled dot shows the direction of an individual bird. The average direction ( $a$, arrow at the center of the circle), sample size ( $n$ ), mean vector length ( $r$, length of the arrow), $p$ value of the Rayleigh test, and $95 \%$ confidence interval (lines beside the arrow) are presented. For confidence intervals, solid lines indicate a $p$ value $<0.05$, while dashed lines indicate a $p$ value $>0.05$. Note that wind direction (black bold arrow outside the circle) is the direction from which wind is blowing. In tests at YLE after sunset (d), the data were excluded from the analysis for four birds tested on the same day in the absence of wind

the birds moved northward. The stronger activity intensity of the Great Knots in the north than in the south Yellow Sea might result from the changes in day length and geomagnetic field. Both day length and geomagnetic cues can be used by migratory birds to obtain seasonal and positional information along their migration routes. Thus, the changes in day length and/or geomagnetic cues may trigger the inherited spatio-temporal regulation system that controls the physiology and behaviour of migrating birds (Berthold 1996; Gwinner 1996). Regarding day length cues, captive birds can be induced into the migratory condition by manipulating day length (Landys et al. 2004a). Regarding geomagnetic cues, simulated displacement along the migratory route by artificial changes in the geomagnetic field affects fuel preparation (Fransson et al. 2001; Kullberg et al. 2007; Henshaw et al. 2008) and orientation behaviour (Henshaw et al. 2010) of captive birds and thus may affect their motivation to resume migration. Probably by changing the plasma corticosterone level of the birds, these external cues could trigger the endogenous system to regulate the migratory restlessness (Landys et al. 2004a; Lõhmus et al. 2003). Further controlled experiments with captive birds will be helpful to untangle and validate the regulation mechanism and procedure.

The inherited spatio-temporal programme is considered as a framework within which other factors such as body condition and weather condition could influence 
behaviour (Gwinner 1996). Our results suggest that the departure schedule of Great Knots might be affected mainly by exogenous factors in the south Yellow Sea while by endogenous factors (the spatio-temporal programme itself) in the north Yellow Sea. At CMDT, during tests before sunset when birds exhibited high intensity of migration activity, body mass was unrelated to migratory motivation while wind effect was the only factor significantly affecting the activity intensity of birds. These results suggest that given the relatively less consuming flight and the low time constraint (relative to the flight from the north Yellow Sea to the breeding sites) from the south to the north Yellow Sea (Ma et al. 2013), the Great Knots could wait for beneficial wind conditions to reduce flight costs (Åkesson and Hedenström 2000; Ma et al. 2011). This is consistent to the prediction of optimal migration theory (Alerstam 2011). YLE in the north Yellow Sea, in contrast, is the final pre-breeding refuelling site for Great Knots. Activity intensity of the birds at YLE increased significantly with the date, indicating time pressure may prevail when approaching the end of the migration. Although the arrivals last for over 1 month, the migratory departure of Great Knots is highly synchronised at YLE (Ma et al. 2013; Peng et al. 2015), which suggests birds probably have an inherited departure schedule at this particular refuelling site. Unexpectedly, at YLE, body mass and wind assistance did not exhibit positive influence on migratory motivation, which might be a hint that the spatio-temporal programme predominates the requirement for long-distance migratory flights. As time advances, birds become less selective concerning favourable wind conditions, and they might take-off irrespective of wind conditions (Åkesson and Hedenström 2000) or fuel store in order to catch the narrow time window for reproduction at breeding sites (Tsvey et al. 2007). Departure at unfavorable conditions would have severe impact on the survival and breeding success of birds at breeding sites. Although previous studies have indicated a relationship between fuel stores and migratory restlessness (Able 1977; Yong and Moore 1993; Fusani et al. 2009), a study on glucocorticoid receptor in red knots (Calidris canutus) suggested that hyperphagia and migratory restlessness might be regulated by different mechanisms (Landys et al. 2004b), which could explain the lack of relationship between body mass and activity intensity in the current study. In addition, wind condition was unsuitable for migration during the tests before departure of birds at YLE, which might cause the negative relationship between wind effects and intensity of migratory activity.

On the other hand, the within-day decrease in body mass significantly affected the activity intensity of birds before sunset at YLE, suggesting that migratory birds might be sensitive to acute changes in body condition and might adjust their behaviour accordingly. In songbirds, body mass loss could reduce the plasma cortisosterone concentration of birds, and thus influence their activity intensity and orientation performance (Lõhmus et al. 2003). Birds would prefer stopover possibility ahead along the route if they were lacking body mass gain within the capture day, and they tended to select a more direct route without stopover opportunity if they had exhibited a faster rate of within-day mass gain (Covino and Holberton 2011). The results of the current study are consistent with the songbird studies in that the reduced migratory activity intensity of Great Knots possibly indicated their tendency of stay for some time when their body mass decreased during captive period.

\section{Orientation}

The Great Knots did not demonstrate a seasonally appropriate migratory direction in the orientation funnels in this study. Instead, they demonstrated a unique windrelated orientation, which to our knowledge has not been previously reported by orientation experiments. Although reverse migration has been reported in lean birds looking for a refuelling site (Åkesson et al. 1996a; Sandberg et al. 2002; Deutschlander and Muheim 2009), we suspect that the Great Knots headed SE in the funnels in response to the wind rather than to a food shortage for the following reasons. First, flying in a reverse direction to obtain food seems unlikely at both CMDT and YLE. The Yangtze estuary is the first major stopover site after an energy consuming transoceanic flight (Battley et al. 2000), and there are few or no high-quality sites for refuelling to the southeast of the estuary. At the same time, there is superabundant high-quality food at the YLE (Choi et al. 2017). Thus, the Great Knots have no reason to engage in a reverse flight if they need to increase body stores quickly. Second, the length of the mean vector and the $p$ value of the Rayleigh test increased after the individual directions were shifted in relation to wind, which suggests that orienting against the wind in the funnel is not a randomly directed escape behaviour. Third, relative to passerines and small-sized shorebirds (e.g., dunlins, sharp-tailed sandpipers, and sanderlings) that have previously been studied in orientation experiments (Sandberg and Gudmundsson 1996; Gudmundsson and Sandberg 2000; Grönroos et al. 2010), Great Knots are larger and heavier and thus require more lift to climb when takingoff (Hedenström and Alerstam 1992). The headwind could increase the relative airspeed at the wings and thereby help generate sufficient lift (Norberg and Norberg 1971). Great Knots have been observed to face into the wind when taking-off in the field at CMDT and YLE (ZJ Ma, personal observation). Most former orientation studies focused on small-sized birds. Further studies with 
larger birds under various wind conditions could be helpful to clarify the impact of body size on the response of birds to wind conditions.

Because shorebirds often perform circling flights before departure (Alerstam et al. 1990; Piersma et al. 1990), the departing flocks might make their migration decision after they have detected favourable wind condition during the tentative flights, and their final orientation decision might also be made after take-off. On the other hand, although orientation of naive birds may correspond to their migratory route and direction based on endogenous programs (Berthold 1996), adult birds like those tested in the current study might respond to factors in addition to the endogenous programs. They might be more experienced in drifting with the wind and compensating later when coming across more favourable wind or when approaching the destination (Liechti 2006), which would make their orientation at the beginning of the flight more flexible.

Whether migrating or moving locally, Great Knots must first take-off. Given their habit of taking-off against wind, the relationship between directional preference of scratches in the funnels and migration decision might be not as pronounced in Great Knots as in songbirds and small-sized shorebirds. It follows that the directional preference of scratches in funnels may not be directly related to body condition, date, wind condition, and other external factors as might be expected if the takeoff direction was the same as the migration direction. This probably explains why we did not find any clearcut relationships between directional choices and these factors when comparing the grouped data (Additional file 1: Table S2, S3). However, the proportion of individuals exhibiting orientation different from random was higher at YLE than at CMDT (Table 1), which suggests a stronger motivation for migration at the final pre-breeding stopover site than others.

\section{Additional file}

\footnotetext{
Additional file 1. Table S1: Model selection for predicting the activity intensity of Great Knots during northward migration at two stopover sites, Chongming Dongtan Nature Reserve (CMDT) in the south Yellow Sea and Yalujiang Estuarine Wetland Nature Reserve (YLE) in the north Yellow Sea. Table S2: Comparison of shifted directions of Great Knots grouped by cloud cover, seasonal timing, wind effect, body mass, tide condition, and restlessness at two stopover sites, Chongming Dongtan Nature Reserve (CMDT) in the south Yellow Sea and Yalujiang Estuarine Wetland Nature Reserve (YLE) in the north Yellow Sea. Table S3: Comparison of natural directions of Great Knots grouped by cloud cover, seasonal timing, wind effect, body mass, tide condition, and restlessness at two stopover sites, Chongming Dongtan Nature Reserve (CMDT) in the south Yellow Sea and Yalujiang Estuarine Wetland Nature Reserve (YLE) in the north Yellow Sea.
}

\section{Authors' contributions}

$\mathrm{ZM}$ and $\mathrm{NH}$ designed the experiment, $\mathrm{NH}, \mathrm{QZ}$, and ZM conducted fieldwork, $\mathrm{NH}, \mathrm{SA}$, and QZ analysed the data, $\mathrm{NH}$ led the writing with contribution from SA, QZ, and ZM. All authors read and approved the final manuscript.

\section{Author details}

${ }^{1}$ Coastal Ecosystems Research Station of Yangtze River Estuary, Ministry of Education Key Laboratory for Biodiversity Science and Ecological Engineering, Institute of Biodiversity Science, Fudan University, Shanghai 200433, China. ${ }^{2}$ Centre for Animal Movement Research, Department of Biology, Lund University, Ecology Building, 22362 Lund, Sweden.

\section{Competing interests}

The authors declare that they have no competing interests.

\section{Acknowledgements}

We thank the Chongming Dongtan Nature Reserve and the Yalu Estuarine Wetland Nature Reserve for their support for our fieldwork. We thank Ran Feng, Xuan Zhang, and Jing Qian for helping with the orientation experiments, and Chi-Yeung Choi, Qingquan Bai, David Melville, Hebo Peng, Qiang Ma, Wenjie Xue, Wei Wu, Xuesong Feng, Hongxi Zang, Weiguo Jin, and Shifu Guo for their assistance in the field. We thank Xiao Xie for helping with data analysis. The writing of the manuscript was completed at Lund University with the help from members of the Animal Navigation Lab and the Centre for Animal Movement Research (both headed by S. Åkesson). This study was financially supported by the National Basic Research Program of China (2013CB430404), National Natural Science Foundation of China (31071939 and 31572280), and the State Scholarship Fund of China (201206100060). S. Åkesson is supported by a Project Grant (621-2010-5584) from the Swedish Research Council and a Linnaeus grant to the Centre for Animal Movement Research (CAnMove) from the Swedish Research Council (349-2007-8690) and Lund University.

\section{Ethical standards}

Birds were captured on the intertidal flat with clapnets as part of conventional shorebird banding program organized by the management office of local nature reserves. All the fieldwork was conducted with the permission and support of the local nature reserves and strictly complied with the requirement of Chinese Wild Animal Protection Law.

\section{Publisher's Note}

Springer Nature remains neutral with regard to jurisdictional claims in published maps and institutional affiliations.

Received: 15 February 2017 Accepted: 31 July 2017

Published online: 11 August 2017

References

Able KP. The orientation of passerine nocturnal migrants following offshore drift. Auk. 1977:94:320-30.

Able KP. Common themes and variations in animal orientation systems. Am Zool. 1991;31:157-67

Åkesson S. Do passerines captured at an inland ringing site perform reverse migration in autumn? Ardea. 1999;87:129-38.

Åkesson S, Sandberg R. Migratory orientation of passerines at dusk, night and dawn. Ethology. 1994:98:177-91.

Åkesson S, Bäckman J. Orientation in pied flycatchers: the relative importance of magnetic and visual information at dusk. Anim Behav. 1999:57:819-28.

Åkesson S, Hedenström A. Wind selectivity of migratory flight departures in birds. Behav Ecol Sociobiol. 2000:47:140-4

Åkesson S, Karlsson L, Walinder G, Alerstam T. Bimodal orientation and the occurrence of temporary reverse bird migration during autumn in south Scandinavia. Behav Ecol Sociobiol. 1996a;38:293-302.

Åkesson S, Alerstam T, Hedenström A. Flight initiation of nocturnal passerine migrants in relation to celestial orientation conditions at twilight. J Avian Biol. 1996b;27:95-102. 
Alerstam T. Optimal bird migration revisited. J Ornithol. 2011;152(Suppl 1):5-23.

Alerstam T, Gudmundsson GA, Jönsson PE, Karloson J, Linström Å. Orientation, migration routes and flight behaviour of Knots, Turnstones and Brant Geese departing from Iceland in spring. Arctic. 1990;43:201-14.

Bai QQ, Chen JZ, Chen ZH, Dong GT, Dong JT, Dong WX, Fu YQ, Han YX, Lu G, Li J, Liu Y, Lin Z, Meng DR, Martinez J, Ni GH, Shan K, Sun RJ, Tian SX, Wang FQ, Xu ZW, Yu RD, Yang J, Yang ZD, Zhang L, Zhang M, Zeng XW. Identification of coastal wetlands of international importance for waterbirds: a review of China Coastal Waterbird Surveys 2005-2013. Avian Res. 2015;6:12.

Bamford M, Watkins D, Bancroft W, Tischler G, Wahl J. Migratory shorebirds of the East Asian-Australasian Flyway: population estimates and internationally important sites. Canberra: Wetlands International, Oceania; 2008.

Batschelet E. Circular statistics in biology. London: Academic Press; 1981.

Battley PF. The northward migration of Arctic waders in New Zealand: departure behaviour, timing and possible migration routes of red knots and bar-tailed godwits from Farewell Spit. North-West Nelson. Emu. 1997:97:108-20.

Battley PF, Piersma T, Dietz MW, Tang S, Dekinga A, Hulsman K. Empirical evidence for differential organ reductions during trans-oceanic bird flight. Proc R Soc B. 2000;267:191-6.

Berthold P. Control of bird migration. London: Chapman and Hall; 1996.

Burnham KP, Anderson DR. Model selection and inference: a practical information-theoretic approach. 2nd ed. New York: Springer; 2002.

Choi CY, Gan XJ, Ma Q, Zhang KJ, Chen JK, Ma ZJ. Body condition and fuel deposition patterns of calidrid sandpipers during migratory stopover. Ardea. 2009;97:61-70.

Choi CY, Battley PF, Potter MA, Ma ZJ, Melville DS, Sukkaewmanee P. How migratory shorebirds selectively exploit prey at a staging site dominated by a single prey species. Auk. 2017;134:76-91.

Covino KM, Holberton RL. The influence of energetic condition on flight initiation and orientation of migratory songbirds in the Gulf of Maine region. Auk. 2011;128:313-20.

Deutschlander ME, Muheim R. Fuel reserves affect migratory orientation of thrushes and sparrows both before and after crossing an ecological barrier near their breeding grounds. J Avian Biol. 2009:40:85-9.

Emlen ST, Emlen JT. A technique for recording orientation of captive birds. Auk. 1966;83:361-7.

Fransson T, Jakobsson S, Johansson P, Kullberg C, Lind J, Vallin A. Magnetic cues trigger extensive refuelling. Nature. 2001;414:35-6.

Fusani L, Cardinale M, Carere C, Goymann W. Stopover decision during migration: physiological conditions predict nocturnal restlessness in wild passerines. Biol Lett. 2009:5:302-5.

Grönroos J, Muheim R, Åkesson S. Orientation and autumn migration routes of juvenile sharp-tailed sandpipers at a staging site in Alaska. J Exp Biol. 2010;213:1829-35.

Gudmundsson GA, Sandberg R. Sanderlings (Calidris alba) have a magnetic compass: orientation experiments during spring migration in Iceland. J Exp Biol. 2000;203:3137-44.

Gwinner E. Circadian and circannual programmes in avian migration. J Exp Biol. 1996;99:39-48.

Hedenström A, Alerstam T. Climbing performance of migrating birds as a basis of estimating limits for fuel-carrying capacity and muscle work. J Exp Biol. 1992;164:19-38.

Henshaw I, Fransson T, Jakobsson S, Kullberg C. Geomagnetic field affects spring migratory direction in a long distance migrant. Behav Ecol Sociobiol. 2010;64:1317-23.

Henshaw I, Fransson T, Jakobsson S, Lind J, Vallin A, Kullberg C. Food intake and fuel deposition in a migratory bird is affected by multiple as well as single-step changes in the magnetic field. J Exp Biol. 2008;211:649-53.

Kullberg C, Henshaw I, Jakobsson S, Johansson P, Fransson T. Fuelling decisions in migratory birds: geomagnetic cues override the seasonal effect. Proc $R$ Soc B. 2007;274:2145-51.

Liechti F. Birds: Blowin' by the wind? J Ornithol. 2006;147:202-11.

Landys MM, Wingfield JC, Ramenofsky M. Plasma corticosterone increases during migratory restlessness in the captive white-crowned sparrow Zonotrichia leucophrys gambelli. Horm Behav. 2004a;46:574-81.
Landys MM, Piersma T, Ramenofsky M, Wingfield JC. Role of the low-affinity glucocorticoid receptor in the regulation of behavior and energy metabolism in the migratory red knot Calidris canutus islandica. Physiol Biochem Zool. 2004b;77:658-68.

Lisovski S, Gosbell K, Hassell C, Minton C. Tracking the full annual-cycle of the great knot Calidris tenuirostris, a long-distance migratory shorebird of the East Asian-Australasian Flyway. Wader Study. 2016;123:177-89.

Lõhmus M, Sandberg R, Holberton RL, Moore FR. Corticosterone levels in relation to migratory readiness in red-eyed vireos (Vireo olivaceus). Behav Ecol Sociobiol. 2003;54:233-9.

Ma ZJ, Hua N, Zhang X, Guo HQ, Zhao B, Ma Q, Xue WJ, Tang CD. Wind conditions affect stopover decisions and fuel stores of shorebirds migrating through the south Yellow Sea. Ibis. 2011;153:755-67.

Ma ZJ, Hua N, Peng HB, Choi CY, Battley PF, Zhou QY, Chen Y, Ma Q, Jia N, Xue WJ, Bai QQ, Wu W, Feng XS, Tang CD. Differentiating between stopover and staging sites: functions of the southern and northern Yellow Sea for long-distance migratory shorebirds. J Avian Biol. 2013;44:504-12.

Mouritsen H. Redstarts, Phoenicurus phoenicurus, can orient in a true-zero magnetic field. Anim Behav. 1998;55:1311-24.

Mouritsen H, Feenders G, Hegemann A, Liedvogel M. Thermal paper can replace typewriter correction paper in Emlen funnels. J Ornithol. 2009;150:713-5.

Muheim R, Åkesson S, Alerstam T. Compass orientation and possible migration routes of passerine birds at high arctic latitude. Oikos. 2003;103:341-9.

Newton I. The migration ecology of birds. London: Academic Press; 2008.

Norberg RÅ, Norberg UM. Take-off, landing, and flight speed during fishing flights of Gavia stellata (Pont.). Ornis Scand. 1971;2:55-67.

Peng HB, Hua N, Choi C, Melville DS, Gao Y, Zhou QY, Chen Y, Xue WJ, Ma Q, Wu W. Adjusting migration schedules at stopping sites: time strategy of a long-distance migratory shorebird during northward migration. J Ornithol. 2015;156:191-9.

Piersma T, Jukema J. Budgeting the flight of a long distance migrant: changes in nutrient levels of Bar-tailed Godwits at successive spring staging sites. Ardea. 1990;78:315-37.

Piersma T, Zwarts L, Bruggemann JH. Behavioural aspects of the departure of waders before long-distance flights: flocking, vocalizations, flight paths and diurnal timing. Ardea. 1990;78:157-84.

Prater AJ, Marchant JH, Vuorinen J. Guide to the identification and ageing of Holarctic waders. BTO field guide 17. Tring: British Trust for Ornithology; 1977.

Richardson WJ. Northeastward reverse migration of birds over Nova Scotia, Canada, in autumn. Behav Ecol Sociobiol. 1982;10:193-206.

Sandberg R. Stored fat and the migratory orientation of birds. In: Berthold P, Gwinner E, Sonnenschein E, editors. Avian migration. Berlin: Springer; 2003. p. 515-25.

Sandberg R, Gudmundsson GA. Orientation cage experiments with dunlins during autumn migration in Iceland. J Avian Biol. 1996;27:183-8.

Sandberg R, Pettersson J, Alerstam T. Why do migrating robins, Erithacus rubecula, captured at two nearby stopover sites orient differently? Anim Behav. 1988;36:865-76.

Sandberg R, Moore FR, Bäckman J, Lõhmus M. Orientation of nocturnally migrating Swainson's Thrush at dawn and dusk: importance of energetic condition and geomagnetic cues. Auk. 2002;119:201-9.

Tsvey A, Bulyuk VN, Kosarev V. Influence of body condition and weather on departures of first-year European robins, Erithacus rubecula, from an autumn migratory stopover site. Behav Ecol Sociobiol. 2007;61:1665-74.

Tulp I, McChesney S, de Goeij P. Migratory departures of waders from northwestern Australia: behaviour, timing and possible migration routes. Ardea. 1994;82:201-21.

Yong W, Moore FR. Relation between migratory activity and energetic condition among thrushes (Turdinae) following passage across the Gulf of Mexico. Condor. 1993;95:934-43. 